Introduction Clinical Research Nurses (CRN) are registered nurses who are employed in industry, charitable and academic healthcare institutions working as part of an infrastructure to support the conduct of clinical research. The CRN role is a well-established role within the United States healthcare system and the role is seen as pivotal when responding to the clinical needs of the participant alongside ensuring the integrity of the research study. Hastings et al (2012) highlight that despite the fact that the CRN role is widely recognized as being an important part of the participant's research journey there, remains a lack of clarity surrounding the standardization and definition of the CRN role.

Aim The purpose of this study is to improve understanding and describe the impact of the role of the clinical research nurse (CRN) on the paediatric participant experience while on a research trial.

Methods This qualitative study used focus groups to explore CRNs, perception of the clinical research nurses impact is on the paediatric participant experience while on a research trial. Data was synthesised using thematic analysis (Braun \& Clark 2006).

Findings Data was collected from 3 sites with a total of 7 focus groups and 33 Participants.

Research Nurses identified 4 key areas of impact (staff, organization, external and participant\& family) with subsequent themes incl compassionate care, family centered care, safety, advocacy.

Conclusions This work clearly demonstrates the CRN value and the impact they have on the participant experience. It highlights the key role nurses play in ensuring participant safety and the responsibilities this entails. This work will enhance the understanding of the role of the CRN and their impact. This will support workforce planning and be used to continually develop and improve the paediatric research participant experience.

\section{ESTABLISHING A CHIEF NURSE JUNIOR FELLOW PROGRAMME @ GOSH}

Lorraine Hodsdon. Great Ormond Street Hospital

\subsection{6/archdischild-2020-gosh.35}

Background This initiative was established as part of a 2019 Florence Nightingale Leadership Scholarship, leadership project. Following discussions with the Chief Nurse it was agreed that the project would focus on engaging and developing our band 5 nursing work force. The Chief Nurse Junior Fellow Programme aligns with the recruitment and retention strategy and the GOSH People Strategy.

Aim To introduce a Chief Nurse Junior Fellowship (CNJF) Programme to GOSH. The programme was open to all band 5 nurses who are at the end of their 18 month rotation programme or band 5 nurses new to GOSH with similar post registration experience.

The programme provides an opportunity for band5 nurses to develop their leadership skills and gain an insight into the senior nursing leadership roles within an NHS organisation through working alongside a senior nurse. In addition it supports a cohort of nurses with the development and acquisition of new leadership skills that they are able to take back into the clinical environment and share with their colleagues.
Method In collaboration with the GOSH Learning Academy a 12 month Chief Nurse Junior Fellow Programme was develop, providing 23 hrs per month protected time for participation along with support from senior nurse mentors across the organisation.

The programme consisted of 3 elements

- Taught Element

- Shadow 'Insight' Opportunities

- Undertake an evidence-based QI project linked to an organisational priority

Outcome 6 Chief Nurse Junior Fellows were appointed in May 2020 and the programme commenced in September 2020. To date they have attended 3 taught study days alongside regular mentor meetings to establish their QI project focus. Quarterly QI progress reports will be submitted alongside pre and post programme leadership evaluations.

\section{FEASIBILITY, ACCEPTABILITY, AND IMPACT OF A COMPUTER-GUIDED GROWTH MINDSET SINGLE- SESSION INTERVENTION FOR CHILDREN AND YOUNG PEOPLE WITH CHRONIC ILLNESS: A MIXED METHODS STUDY}

${ }^{1}$ Brian Chi Fung Ching, ${ }^{1}$ Sophie Bennett, ${ }^{2}$ Sophie Allen, ${ }^{1}$ Isobel Heyman, ${ }^{3}$ Nicola Morant, ${ }^{1}$ Roz Shafran. ${ }^{1}$ Great Ormond Street Hospital and University College London; ${ }^{2}$ Great Ormond Street Hospital; ${ }^{3}$ University College London

\subsection{6/archdischild-2020-gosh.36}

Digital single-session interventions (SSIs) may be an accessible treatment option for children and young people with symptoms of depression and anxiety during COVID-19. The growth mindset SSI is an evidence-based digital SSI designed to enhance beliefs about the malleability of one's traits and problems. A mixed methods case series design was used to assess the feasibility and acceptability of a growth mindset SSI in patients on waiting lists for treatment with the Psychological Medicine team at Great Ormond Street Hospital. It was delivered as an online CAMHS appointment, as part of the treatment offered by the team. Recruitment, treatment completion, and retention rates were collected, and semi-structured interviews were conducted at post-treatment. Preliminary data on the intervention's impact on symptoms of depression and anxiety, perceived control, and personality mindset were collected. Quantitative measures were collected at baseline, posttreatment, and 1-month follow-up. Recruitment and treatment completion rates were high, but retention was moderate. Data from quantitative measures $(n=25)$ showed that patients experienced significantly improved personality mindset (Cohen's $d=0.56$ ) from baseline to post-treatment but not at 1-month follow-up. Changes in symptoms of depression and anxiety and perceived control were non-significant at post-treatment and 1-month follow-up. From the interview data $(\mathrm{n}=17)$, three clusters of themes were identified: overall accessibility and interest, specific components of intervention, and potential impact of intervention. Many patients found learning about neuroplasticity and hearing stories from other young people interesting and instilled a hope for change. Findings suggest the growth mindset SSI is feasible and acceptable for children and young people with chronic illness. The appointment's online format was welcomed by patients and families during the pandemic. The little-to-no 
therapist input suggests the potential widespread use for services in the hospital. Future research and clinical implications are highlighted for implementation and outcomes.

\section{\begin{tabular}{|l|l}
\hline 37 & HYPOXIC CHALLENGE TESTING IN INFANTS; WHO IS
\end{tabular} RECOMMENDED TO FLY WITH SUPPLEMENTAL OXYGEN?}

${ }^{1}$ Mollie Riley, ${ }^{1}$ Emma Fettes, ${ }^{1}$ Aidan Laverty, ${ }^{2}$ Paul Aurora. ${ }^{1}$ Great Ormond Street Hospital; ${ }^{2}$ Great Ormond Street Hospital and University College London

10.1136/archdischild-2020-gosh.37

Infants with a history of neonatal chronic respiratory problems may demonstrate hypoxaemia when at in-flight oxygen levels, despite normal sea-level oxygen requirements. The British Thoracic Society recommend these infants have hypoxic challenge testing (HCT) before air travel, $\mathrm{SpO}_{2}<85 \%$ is recommended as a threshold below which in-flight oxygen is required and 'paediatrician discretion should be used when $\mathrm{SpO}_{2}$ between $85-90 \%$ and, where there is doubt, the doctor should err on the side of caution.'

Aim To establish how many infants fell into each of the threshold categories during HCT; $\mathrm{SpO}_{2}<85 \%$, >90\%, 85$90 \%$ and which of these patients were recommended to fly \pm supplemental oxygen $\left(\operatorname{suppO} \mathrm{O}_{2}\right)$.

Methods Our HCT protocol for infants is 20 minutes in 15\% $\mathrm{FiO}_{2}$ within a body plethysmograph. $\mathrm{SpO}_{2}$ is monitored throughout and $\operatorname{suppO}_{2}$ administered via nasal cannula if $\mathrm{SpO}_{2}<85 \%$. If after 20 minutes $\mathrm{SpO}_{2}$ has remained $>85 \%$ but $<90 \%$ then suppO ${ }_{2}$ is titrated for 5 minutes. We reviewed data collected from infants (aged $<1$ year) whom had HCT between March 2017- January 2020.

Results Data collected from 65 infants, median age 27.6 weeks (range 5 to 51.6), 37 were male. None were receiving suppO ${ }_{2}$ in room air prior to testing; all had baseline $\mathrm{SpO}_{2} \geq 96 \%$. In 40 infants, $\mathrm{SpO}_{2}$ did not dip to $<90 \% . \mathrm{SpO}_{2}$ dropped to $<85 \%$ in 16 infants, requiring administration of suppO $\mathrm{P}_{2} .9$ infants required extended protocol due to $\mathrm{SpO}_{2} 85-90 \%$. SuppO $\mathrm{O}_{2}$ corrected $\mathrm{SpO}_{2}$ in all to baseline levels.

In the 85-90\% category, all 9 infants were advised by their clinician to use $\operatorname{suppO} \mathrm{O}_{2}$ for air travel.The flight times in this subgroup ranged from 90 to 450 minutes.

Conclusion Infants with baseline $\mathrm{SpO}_{2} \geq 96 \%$ may still exhibit $\mathrm{SpO}_{2}$ desaturation during HCT. We found all paediatricians recommended in-flight oxygen for infants with $\mathrm{HCT} \mathrm{SpO}_{2}$ $<90 \%$ regardless of flight duration.

\section{ADAPTING THE DELIVERY OF PSYCHOLOGICAL INTERVENTION IN A CHILDREN'S HEADACHE SERVICE IN RESPONSE TO COVID-19}

Caitlin Black, Chloe Charnick, Sophie Mitchell, Nikolaos Sarras, Elena Bantoft, Hayley Bullock. Great Ormond Street Hospital

10.1136/archdischild-2020-gosh.38

Objectives Psychological intervention forms part of a multidisciplinary approach to the treatment of headache conditions in childhood. Within the Children's Headache Service at Great Ormond Street Hospital many children and young people typically access this in the format of a group intervention. In response to the impact of COVID-19 on service provision, the group materials were adapted into an alternative lowintensity psychological intervention format to be offered via individual video appointments.

Methods The group materials were reviewed and adapted into a six session guided self-help intervention. Session material was underpinned by an evidence-based approach utilising a CBT model, which forms the current basis of psychological headache intervention. This intervention was offered to young people who were originally referred to the group, and who subsequently agreed to the alternative guided self-help intervention. Goal-based outcome measures were used to monitor progress during the course of the intervention. The materials were reviewed by the practitioners providing the intervention and the young people accessing the intervention were also asked for feedback to enable continued adaptation and development based on experience.

Results Initial results suggest that a guided self-help intervention via video appointments is an accepted low-intensity psychological intervention by young people with headache conditions.

Conclusion Service found a way to adapt support for young people with headache conditions in the face of exceptional circumstances due to Covid-19. This has implications for the future provision of interventions and improves accessibility for the young people referred to our service who may otherwise be limited by financial or geographical means.

\section{PEERS - POST EVENT EVALUATION REFLECTION AND SUPPORT}

Mike Stylianou, Ciara McMullin, Rajan Saini. Great Ormond Street Hospital

\subsection{6/archdischild-2020-gosh.39}

The NHS is the largest employer in the United Kingdom, the largest employer in Europe and the fifth largest employer in the World employing over 1.7 million people. Great Ormond Street Hospital employs over 4200 of these individuals.

Many teams and Industries support their teams by debriefing following significant negative events and indeed some teams debrief all events.

Healthcare teams are unique when compared to teams in other industries. Health Care teams differ in Gender, age, culture, ethnicity, cognitive ability, experience and training and specialty. The spectrum of a healthcare team may range from student nurse to professor all working with a common same focus -'the Patient'.

Health care teams are Heterogeneous unlike the Homogenous nature of teams such as the Military or Aerospace which are for example, predominantly male, similar backgrounds and similar ages.

PEERS recognises and responds to needs diverse of individuals and teams that make up our healthcare system.

The aim of PEERS meetings is to provide support for colleagues, assisting them to explore and make sense of and process common reactions to potentially stressful events.

The PEERS approach seeks, nurture resilience, vulnerability and wellbeing through supported reflection by creating a safe space where individuals can speak openly and honestly, and be heard without bias, judgment or threat.

The fundamental objective is to foster, support and encourage, personal growth, wellbeing and to encourage 'help-seeking' behaviour, if needed. 